\title{
Lesión de Galeazzi: evolución de la articulación radiocubital distal a largo plazo
}

\author{
P. De Carli ${ }^{(1)}$, A. DonndorfF ${ }^{(2)}$, G. Gallucci ${ }^{(3)}$, J. $^{\text {Boretto }}{ }^{(2)}$, A. Sarme $^{(4)}$, \\ V. ALFIE ${ }^{(2)}$ \\ Instituto de Ortopedia y TRaumatología «CARlos E. Ottolenghi». Sector Cirugía de la Mano Y \\ Miembro Superior. Hospital italiano de Buenos Aires. Argentina \\ (1)JEFE DE SECCIÓN DE CIRUGÍA DE LA MANO Y MIEMBRo SUPERIOR \\ ${ }^{(2)}$ CIRUJANO DE MANO ASOCIADO \\ ${ }^{(3)}$ CiRUJano de Planta \\ ${ }^{(4)}$ BECARIO DE ESPECIALIZACIÓN
}

Correspondencia:

Dr. Pablo De Carli

Potosí 4247, Cap. Fed.

CP 1199. Buenos Aires, Argentina

Tel.: +541147833832

Fax: +5411 49810991

e-mail: pablo.decarli@hospitalitaliano.org.ar

Introducción: Si bien se considera que en las lesiones de Galeazzi es esencial la reducción anatómica del radio para lograr la reducción secundaria del cúbito, existen todavía controversias acerca del tratamiento y pronóstico de la articulación radiocubital distal. El objetivo es evaluar clínica y radiológicamente la evolución a largo plazo de la articulación radiocubital distal en este tipo de lesiones. Material y Métodos: Se analizaron retrospectivamente 9 pacientes con una edad promedio de 46 años, con diagnóstico de lesión de Galeazzi tratados con osteosíntesis con placa desde 1993 a 2001, y con un seguimiento mínimo de 6 años. En el control final, seguimiento promedio de 8 años, se evaluó clínicamente la articulación radiocubital distal en forma objetiva (signo de la tecla, maniobra de compresión, rango de movilidad y fuerza de puño) y subjetiva (escala analógica visual y DASH). Se realizó además una evaluación radiográfica y tomográfica comparativa con el lado contralateral para determinar la presencia de inestabilidad (método de Mino) y artrosis radiocubital (clasificación de Knirk-Jupiter).

Resultados: Clínicamente, todos los pacientes presentaron signo de la tecla positivo, presentando uno sólo dolor leve a la compresión. El rango de movi-
Introduction: While in Galeazzi's fracture is well accepted that stable and optimal reduction of the radius fracture is indispensable to achieve a secondary reduction of the ulna, the treatment and prognostic of the distal radioulnar joint may still be controversial. The aim of this study is to evaluate the long-term functional and radiographic outcomes of the distal radioulnar joint in this type of lesions.

Material and Method: Nine patients with an average age of 46 years presenting a Galeazzi fracture treated with ORIF of the radius between 1993 and 2001 were retrospectively evaluated, minimum follow-up: 6 years. At final control, average: 8 years, the distal radioulnar joint was objectively (piano key test, compression maneuver, wrist range of motion, and grip strength) and subjectively (visual analogic scale and DASH questionnaire). Radiographs and computerized tomography were performed to determinate the presence of distal radioulnar instability (method of Mino) and/or arthrosis (classification of Knirk and Jupiter).

Results: Clinically, all patients presented a positive piano key test, but only one of them report mild pain with the compression maneuver. No statistical differences were observed in range of motion bet- 
lidad no mostró diferencia significativa y la fuerza de puño fue $78 \%$ comparada con el contralateral. La escala analógica visual promedio fue de 0,8 sobre 10, y el DASH de 8 (escala 0 a 100). Si bien 2 casos presentaron radiológicamente una subluxación dorsal leve del cúbito, en ningún caso se observó evidencia de inestabilidad en la evaluación tomográfica. En 6 casos no se observó artrosis, en 1 caso artrosis leve, y en 2 casos artrosis moderada. No se observó correlación entre inestabilidad y artrosis. Los resultados obtenidos sugieren que luego de una adecuada reducción de la fractura del radio en lesiones de Galeazzi la evolución clínica y radiológica a largo plazo de la articulación radiocubital distal es favorable, presentando una inestabilidad leve bien tolerada, y sin cambios artrósicos graves.

Palabras clave: fractura de radio distal, articulación radiocubital distal, fractura luxación de Galeazzi. ween injured-contralateral wrists, and mean comparative grip strength was $78 \%$. The average visual analogic scale and DASH were 0.8 (0 to 10) and 8 (0 to 100), respectively. Although 2 cases presented radiologically a mild dorsal subluxation of the ulna, none of the 9 patients presented tomographically evidence of instability. In 6 cases no arthrosis were observed, in 1 case a mild arthrosis, and in 2 cases a moderate arthrosis. No correlation was observed between instability and arthrosis.

Conclusions: Our findings suggest that after a stable and optimal reduction and a rigid internal osteosynthesis of the radius fracture in Galeazzi's fractures the long-term prognostic of the distal radioulnar joint is favorable, presenting the patients a mild well tolerated instability, without degenerative changes.

Key words: distal radius fracture, distal radioulnar joint, Galeazzi fracture-dislocation.

Rev. Iberam. Cir. Mano - Vol. 35 • Núm. 2 - Noviembre 2007 (71-81)

\section{INTRODUCCIÓN}

T a lesión de Galeazzi consiste en la luxación traumática de la articulación radiocubital distal (RCD) asociada a la fractura de la diafisis radial en la unión de su tercio medio o inferior. Si bien fue descrita inicialmente por Cooper en $1822^{1}$, esta lesión recibió su nombre luego de que Ricardo Galeazzi presentara, en la Sociedad de Cirugía de Lombardía, 18 casos en $1934^{2}$. Representa una lesión infrecuente, con una incidencia del 6 al $7 \%$ de todas las fracturas del antebrazo de acuerdo con algunos autores ${ }^{3,4}$.

Se considera ampliamente en la literatura que la reducción anatómica del radio es esencial para lograr la reducción secundaria del cúbito ${ }^{3-6}$. Sin embargo, basados en la literatura revisada, se encuentra poca información acerca del pronóstico de la articulación radiocubital distal en este tipo de lesiones ${ }^{6-9}$. De esta manera, existen todavía controversias relacionadas con el tratamiento en agudo de la articulación RCD.

El objetivo de este trabajo es evaluar la evolución clínica y radiológica a largo plazo de la articulación RCD reducida indirectamente luego de la reducción abierta y fijación interna de la fractura del radio.

\section{MATERIAL Y MÉTODO}

Entre enero de 1993 y mayo de 2001, se trataron quirúrgicamente en forma consecutiva 25 pacientes con diagnóstico de lesión aguda de Galeazzi. Se consideraron como criterios de inclusión para nuestro estudio: 1) pacientes mayores de 18 años (epífisis cerrada); 2) lesiones agudas cerradas, no operadas previamente; 3 ) fractura radial consolidada, tratada inicialmente con reducción abierta y osteosíntesis con placa, con una reducción anatómica (sin angulación, rotación ni acortamiento); 4) reducción secundaria del cúbito tratada en forma incruenta mediante inmovilización de la prono-supinación con yeso braquiopalmar por 3 semanas; 5) más de 6 años de seguimiento.

De este modo, se excluyeron 4 casos que fueron tratados con otros métodos de fijación del radio, 1 caso que fue tratado con cirugía de rescate en la articulación radio-cubital inferior, y otro caso que presentó una mala consolidación del radio con angulación de $30^{\circ}$. Otros 10 casos se descartaron por ser operados inicialmente en otro centro y requirieron una segunda cirugía.

El grupo de estudio consistió entonces en 9 pacientes, 6 hombres y 3 mujeres, con una edad 


\section{Tabla I - CaRACterísticas Individuales de los PaCientes}

\begin{tabular}{|c|c|c|c|c|c|c|}
\hline CASO & $E D A D$ & GÉNERO & OCUPACION & $\begin{array}{l}\text { MIEMBRO } \\
\text { LESIONADO }\end{array}$ & $\begin{array}{c}\text { MIEMBRO } \\
\text { DOMTNANTE }\end{array}$ & $\begin{array}{c}\text { SEGUTMENTO } \\
\text { (meses) }\end{array}$ \\
\hline 1 & 79 & $\mathrm{~F}$ & Ama de casa & Der. & Der. & 149 \\
\hline 2 & 49 & M & Enfermero & Izq. & Der. & 100 \\
\hline 3 & 36 & F & Ama de casa & Der. & Der. & 76 \\
\hline 4 & 39 & M & Oficinista & Izq. & Der. & 84 \\
\hline 5 & 33 & M & Oficinista & Izq. & Der. & 85 \\
\hline 6 & 25 & M & Psicólogo & Izq. & Izq. & 117 \\
\hline 7 & 51 & M & Fotógrafo & Izq. & Der. & 72 \\
\hline 8 & 49 & $\mathrm{~F}$ & Administrativa & Izq. & Der. & 84 \\
\hline 9 & 52 & M & Maestro & Izq. & Der. & 124 \\
\hline
\end{tabular}

promedio de 46 años (rango: 25 a 79). En 3 casos el miembro lesionado fue el dominante. Todos los pacientes presentaron una luxación hacia el dorso del cúbito y fueron operados dentro de la primera semana del traumatismo. En 8 casos se fijó el radio con placas DCP $\left(\right.$ Synthes $^{\circledR}$ ), y en 1 caso con placa LC-DCP $\left(\right.$ Synthes ${ }^{\circledR}$ ). En 8 casos se utilizó un abordaje palmar, y en 1 caso un abordaje dorsal. Todos los pacientes incluidos fueron evaluados en forma retrospectiva clínica y radiológicamente, con un seguimiento promedio de 8 años (rango: 6 a 12). Tabla I.

\section{Evaluación clínica}

Todas las muñecas fueron evaluadas clínicamente de forma bilateral.

La evaluación objetiva de la articulación RCD incluyó: el signo de la tecla (como signo de inestabilidad) en posición neutra, supinación y pronación máximas; y la presencia de dolor durante la movilización pasiva de la muñeca o al realizar una maniobra de compresión lateromedial sobre la articulación RCD. Además se evaluaron los rangos de movilidad de la muñeca goniométricamente, y la fuerza de puño con un dinamómetro (Jamar $)^{10}$.

Por otro lado, se realizó una evaluación subjetiva de cada paciente: la presencia de dolor durante las actividades diarias mediante la escala analógica visual, la función global del miembro lesionado a través del cuestionario DASH (Disabilities of the Arm, Shoulder and Hand $)^{11}$.

\section{Evaluación radiológica}

En todos los pacientes se realizó una evaluación de las radiografías (frente y perfil) preoperatorias, postoperatorias inmediatas, postoperatorias alejadas (en el último control), y de la muñeca contralateral, con el fin de determinar la calidad de la reducción y su mantenimiento en el tiempo, la presencia de inestabilidad y artrosis RCD. Cada radiografía fue rea- 


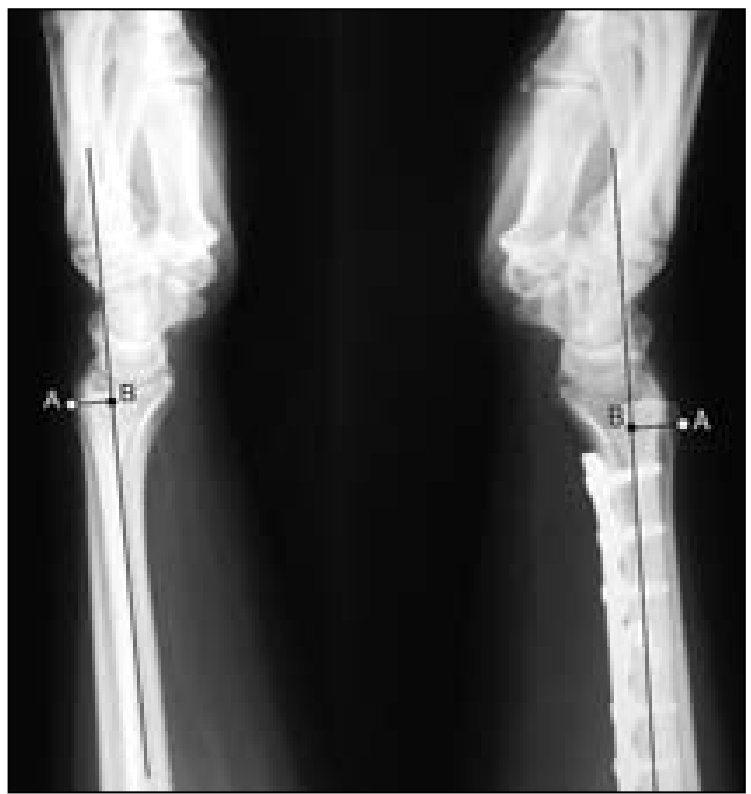

Figura 1. Evaluación de la subluxación del cúbito en radiografías de perfil comparativas midiendo la distancia entre el borde más dorsal del cúbito (A) y el eje del radio $(B)$.

lizada con el hombro en abducción de $90^{\circ}$, codo flexionado $90^{\circ}$, y palma de la mano apoyada sobre el chasis (antebrazo en rotación neutra) para las proyecciones de frente; con el brazo contra el cuerpo (abducción de $0^{\circ}$ ), el codo en flexión de $90^{\circ}$, y la muñeca en posición neutra para las proyecciones de perfil ${ }^{12}$.

En las proyecciones anteroposteriores se evaluó la variante cubital según el método de las perpendiculares, reconocido por Steyers y Blair como el más reproducible ${ }^{13}$.

En las proyecciones de perfil se analizó la presencia de subluxación del cúbito con respecto al radio (Figura 1).

En ambas proyecciones de las radiografías alejadas se evaluó la presencia artrosis RCD comparativamente con la muñeca contralateral, utilizando la clasificación de Knirk y Jupiter (pinzamiento articular, osteofitos marginales, $\mathrm{y}$ esclerosis subcondral) ${ }^{14}$.

Además, se evaluó la inestabilidad y el grado de artrosis RCD tomográficamente en 5 pacientes (4 no aceptaron la realización de la tomografía), comparándola con el miembro contralateral sano. La tomografía axial computerizada (TAC) se realizó en todos los casos colocando al paciente en decúbito ventral sobre la mesa móvil del tomógrafo con los hom-

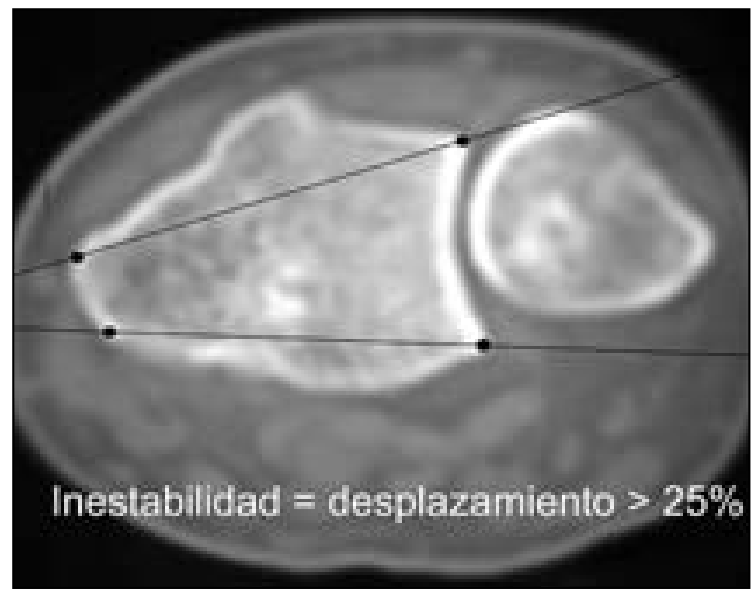

Figura 2. Evaluación de la subluxación cubital mediante tomografía computerizada según el método de Mino $^{15-16}$. Se trazan dos líneas, una a través de los bordes radial y cubital dorsales (A), y otra a través de los bordes radial y cubital palmares (B). En una articulación RCD estable la cabeza del cúbito debería estar entre las dos líneas en la posición neutra de la muñeca.

bros en elevación completa y se colocó una banda elástica en cada muñeca a nivel del tubérculo de Lister. En primera instancia se realizaron cortes en posición neutra, y posteriormente, utilizando la banda elástica como marca, se realizaron los mismos cortes en máxima supinación y máxima pronación. La inestabilidad se evaluó a través del método de Mino et al. ${ }^{15,16}$, el más utilizado en la literatura ${ }^{15-18}$. Se trazan 2 líneas que pasan por los bordes dorsal y palmar del radio (Figura 2). Se considera normal si la cabeza del cúbito se encuentra entre esas 2 líneas en posición neutra de la muñeca. Si la cabeza del cúbito se localiza por fuera de estas líneas, se calcula la proporción de la cabeza que se encuentra palmar o dorsal a ellas. Un desplazamiento mayor al $25 \%$ se considera como evidencia de subluxación. Con el fin de lograr una evaluación dinámica de la estabilidad RCD, se utilizó también este mismo método en pronación y supinación máxima, comparando al mismo tiempo el desplazamiento cubital con la muñeca contralateral. La artrosis se clasificó mediante la misma escala de Knirk y Jupiter para la evaluación radiográfica $^{14}$.

Todas las evaluaciones clínicas y radiológicas fueron realizadas por uno solo de los autores, el cual no estuvo involucrado en el tratamiento inicial y seguimiento de los pacientes. 


\section{Tabla II - Signo DE LA TECLA COMPARATIVO}

\begin{tabular}{|c|c|c|c|c|c|c|}
\hline \multirow{2}{*}{ CASO } & \multicolumn{3}{|c|}{ MIEMBRO LESIONADO } & \multicolumn{3}{|c|}{ MIEMBRO CONTRALATERAL } \\
\hline & neutro & pronación & supinación & neutro & pronación & supinación \\
\hline 1 & si & no & no & no & no & no \\
\hline 2 & si & no & no & no & no & no \\
\hline 3 & si & si & no & si & no & no \\
\hline 4 & si & si & no & sî & no & no \\
\hline 5 & si & si & si & no & no & no \\
\hline 6 & no & no & si & no & no & no \\
\hline 7 & si & no & no & no & no & no \\
\hline 8 & si & no & si & si & no & no \\
\hline 9 & si & si & no & si & no & no \\
\hline
\end{tabular}

\section{Análisis estadístico}

Se realizó un análisis estadístico de los resultados obtenidos mediante la prueba de Student para intentar determinar la presencia o no de diferencias estadísticamente significativas en la movilidad de muñeca y fuerza de puño del miembro lesionado y el contralateral sano. Se consideró como valor significativo una $p<0,05$.

\section{RESULTADOS}

\section{Evaluación clínica}

Al comparar la estabilidad RCD con la muñeca contralateral, todos los pacientes presentaron signo de la tecla en alguna de las 3 posiciones de la muñeca: 4 en posición neutra, 3 en pronación, 2 en supinación, y 1 en las tres posiciones (Tabla II). Sin embargo, uno solo de ellos (caso 3) presentó dolor sobre la articulación RCD durante la compresión manual.
Los rangos de movilidad promedio de la muñeca (comparativos) fueron (Tabla III): flexión $=68^{\circ}\left(76^{\circ}\right)$; extensión $=74^{\circ}\left(75^{\circ}\right)$; desviación radial $=22^{\circ}\left(22^{\circ}\right)$; desviación cubital $=35^{\circ}$ $\left(35^{\circ}\right)$; pronación $=87^{\circ}\left(88^{\circ}\right)$; supinación $=84^{\circ}$ $\left(89^{\circ}\right)$. No se encontraron diferencias significativas en ninguno de los rangos de movilidad entre la muñeca lesionada y la contralateral. La fuerza de puño promedio de la muñeca lesionada fue del $83 \%$ con respecto a la contralateral, con un promedio $27 \mathrm{~kg}$ y $32 \mathrm{~kg}$, respectivamente. Esta diferencia no fue estadísticamente significativa $(p=0,09)$.

En relación a la evaluación subjetiva, la presencia de dolor durante las actividades diarias según la escala analógica visual fue de 0.8 promedio (rango: 0 a 2,5), en una escala de 0 a 10 , en donde 10 equivale al máximo dolor. El test de DASH promedio para evaluar la función fue de 8 (rango: 2,5 a 30,5) en una escala de 0 a 100 (mayor puntuación, peor función). Todos los pacientes demostraron satisfacción con el 


\section{Tabla III - RANGoS DE MOVILIDAD Y FUERZA DE PUÑo COMPARATIVOS}

\begin{tabular}{|c|c|c|c|c|c|c|c|c|c|c|c|c|c|c|}
\hline \multirow[t]{2}{*}{ Caso } & \multicolumn{2}{|c|}{ Flexión } & \multicolumn{2}{|c|}{ Extensión } & \multicolumn{2}{|c|}{ D.R. } & \multicolumn{2}{|c|}{ D.C. } & \multicolumn{2}{|c|}{ Pronación } & \multicolumn{2}{|c|}{ Supinación } & \multicolumn{2}{|c|}{ Fuerza de pun̂o } \\
\hline & L & $\mathrm{s}$ & L & s & $\mathrm{L}$ & $\mathrm{s}$ & L & s & L & S & L & S & L & s \\
\hline 1 & $70^{\circ}$ & $75^{\circ}$ & $55^{\circ}$ & $80^{\circ}$ & $25^{\circ}$ & $20^{\circ}$ & $40^{\circ}$ & $50^{\circ}$ & $80^{\circ}$ & $80^{\circ}$ & $70^{\circ}$ & $90^{\circ}$ & $10 \mathrm{Kgr}$. & $9 \mathrm{Kgr}$. \\
\hline 2 & $75^{\circ}$ & $80^{\circ}$ & $85^{\circ}$ & $75^{\circ}$ & $25^{\circ}$ & $25^{\circ}$ & $35^{\circ}$ & $35^{\circ}$ & $80^{\circ}$ & $90^{\circ}$ & $80^{\circ}$ & $90^{\circ}$ & $34 \mathrm{Kgr}$. & $27 \mathrm{Kgr}$. \\
\hline 3 & $80^{\circ}$ & $70^{\circ}$ & $75^{\circ}$ & $80^{\circ}$ & $25^{\circ}$ & $25^{\circ}$ & $45^{\circ}$ & $45^{\circ}$ & $90^{\circ}$ & $90^{\circ}$ & $90^{\circ}$ & $90^{\circ}$ & $13 \mathrm{Kgr}$. & $20 \mathrm{Kgr}$. \\
\hline 4 & $70^{\circ}$ & $80^{\circ}$ & $85^{\circ}$ & $80^{\circ}$ & $20^{\circ}$ & $25^{\circ}$ & $40^{\circ}$ & $30^{\circ}$ & $90^{\circ}$ & $90^{\circ}$ & $90^{\circ}$ & $90^{\circ}$ & $36 \mathrm{Kgr}$. & $43 \mathrm{Kgr}$. \\
\hline 5 & $60^{\circ}$ & $75^{\circ}$ & $80^{\circ}$ & $80^{\circ}$ & $40^{\circ}$ & $25^{\circ}$ & $30^{\circ}$ & $30^{\circ}$ & $80^{\circ}$ & $80^{\circ}$ & $80^{\circ}$ & $80^{\circ}$ & $28 \mathrm{Kgr}$. & $41 \mathrm{Kgr}$. \\
\hline 6 & $80^{\circ}$ & $80^{\circ}$ & $70^{\circ}$ & $70^{\circ}$ & $20^{\circ}$ & $30^{\circ}$ & $30^{\circ}$ & $30^{\circ}$ & $90^{\circ}$ & $90^{\circ}$ & $90^{\circ}$ & $90^{\circ}$ & $25 \mathrm{Kgr}$. & $25 \mathrm{Kgr}$. \\
\hline 7 & $40^{\circ}$ & $75^{\circ}$ & $65^{\circ}$ & $60^{\circ}$ & $10^{\circ}$ & $10^{\circ}$ & $35^{\circ}$ & $35^{\circ}$ & $90^{\circ}$ & $90^{\circ}$ & $80^{\circ}$ & $90^{\circ}$ & $19 \mathrm{Kgr}$. & $38 \mathrm{Kgr}$. \\
\hline 8 & $70^{\circ}$ & $80^{\circ}$ & $80^{\circ}$ & $80^{\circ}$ & $20^{\circ}$ & $20^{\circ}$ & $30^{\circ}$ & $30^{\circ}$ & $90^{\circ}$ & $90^{\circ}$ & $90^{\circ}$ & $90^{\circ}$ & $21 \mathrm{Kgr}$. & $32 \mathrm{Kgr}$. \\
\hline 9 & $70^{\circ}$ & $70^{\circ}$ & $70^{\circ}$ & 70 & $10^{\circ}$ & $15^{\circ}$ & $30^{\circ}$ & $30^{\circ}$ & $90^{\circ}$ & $90^{\circ}$ & $90^{\circ}$ & $90^{\circ}$ & $35 \mathrm{Kgr}$. & $54 \mathrm{Kgr}$. \\
\hline Prom & $68^{\circ}$ & $76^{\circ}$ & $74^{\circ}$ & $75^{\circ}$ & $22^{\circ}$ & $22^{\circ}$ & $35^{\circ}$ & $35^{\circ}$ & $87^{\circ}$ & $88^{\circ}$ & $84^{\circ}$ & $89^{\circ}$ & $25 \mathrm{Kgr}$. & $32 \mathrm{Kgr}$. \\
\hline$P$ & \multicolumn{2}{|c|}{0,1} & \multicolumn{2}{|c|}{0,75} & \multicolumn{2}{|c|}{1,00} & \multicolumn{2}{|c|}{1,00} & \multicolumn{2}{|c|}{0,35} & \multicolumn{2}{|c|}{0,1} & \multicolumn{2}{|c|}{0,035} \\
\hline
\end{tabular}

D.R.: desviación radial; D.C.: desviación cubital; L: lesionado; S: sano; Kgr: kilogramos; Prom.: promedio.

resultado obtenido y retornaron a sus actividades diarias y laborales previas.

\section{Evaluación radiológica}

En las radiografías preoperatorias (Tabla IV), los pacientes presentaron un acortamiento cubital inicial promedio de 16,6 mm (rango: 14 a 19); en el postoperatorio inmediato la variante cubital promedio fue de $0,2 \mathrm{~mm}$ (rango: -2 a 3). En el último control postoperatorio la variante cubital fue de $0,9 \mathrm{~mm}$ (rango: -2 a 3 ); y en el lado contralateral correspondió a $0,8 \mathrm{~mm}$ (rango: -1 a 3). La diferencia de la variante cubital entre la reducción inmediata y la contralateral sana no fue significativa $(p=0,1)$.

En 2 casos (casos 3 y 8) se observó una subluxación dorsal del cúbito con respecto al radio en las radiografías de perfil, comparándolas con el lado sano. Sin embargo, en la evaluación tomográfica no se logró determinar una subluxación comparativa evidente $(>25 \%)$ en ninguno de los 5 casos evaluados en las 3 posiciones de la muñeca (Tabla V). En 4 casos se observó un leve aumento del desplazamiento palmar del cúbito con respecto al contralateral sano que no superó el 12\% (entre el 3\% y el 12\%) durante la supinación máxima. Un caso presentó una tendencia a un mayor desplazamiento hacia el dorso comparativamente que no superó el $4 \%$ durante la pronación máxima.

En las radiografías postoperatorias obtenidas en el último control, 2 pacientes presentaron artrosis grado II de la clasificación de Knirk y Jupiter ${ }^{14}, 1$ paciente presentó artrosis grado I, y los 6 restantes artrosis grado 0 (sin cambios artrósicos) (Tabla VI). Uno de los dos pacientes que presentaron una artrosis grado II (caso 1) fue una mujer de 79 años que presentaba el mismo grado de artrosis en la muñeca contralateral. Al evaluar la artrosis mediante tomogra- 


\section{Tabla IV - VARIANTE CUBITAL PREOPERATORIO, POSTOPERATORIA INMEDIATA Y ALEJADA}

\begin{tabular}{|c|c|c|c|}
\hline \multirow{2}{*}{ CASO } & \multicolumn{3}{|c|}{ VARLANTE CUBTTAL Lesionada/Sana (mm) } \\
\hline & Rx. Preoperatoria & Rx. Postoperatoria Inmediata & Rx. PostopenatoriaAlejada * \\
\hline$I$ & 16 & $3 / 3$ & 2 \\
\hline 2 & 14 & $-1 /-1$ & 2 \\
\hline 3 & 18 & $-2 /-1$ & 0 \\
\hline 4 & 19 & $1 / 0$ & 1 \\
\hline 5 & 16 & $-2 / 0$ & -2 \\
\hline 6 & 14 & $0 / 0$ & -2 \\
\hline 7 & 19 & $1 / 2$ & 2 \\
\hline 8 & 16 & $2 / 3$ & 2 \\
\hline 9 & 18 & $0 / 1$ & 3 \\
\hline Promedio & 16,6 & $0,2(0,8) p=0,1$ & 0,9 \\
\hline
\end{tabular}

- Radiografias obtenidas en el último control.

fía, 3 casos en los cuales no se observó artrosis radiológicamente (grado 0 ) presentaban signos de artrosis grado I. Al mismo tiempo, 6 de los 9 casos presentaron una calcificación (3 casos) o seudoartrosis (3 casos) en la estiloides cubital.

\section{DISCUSIÓN}

El objetivo del tratamiento de las lesiones de Galeazzi consiste en restaurar la congruencia y estabilidad de la articulación radiocubital distal. Si bien numerosos estudios describen que la reducción anatómica del radio y fijación interna del radio es esencial para lograr una adecuada reducción secundaria del cúbito $^{3-6}$, una buena reducción y fijación radial no asegura la estabilidad RCD. De este modo, no hemos encontrado información concluyente acerca impacto de este tipo de lesiones sobre la articu- lación RCI clínica y radiológicamente a largo plazo.

En un estudio retrospectivo sobre 25 casos con un seguimiento de 6,5 años promedio, Mestdagh et al. reportaron 8 casos con desplazamiento persistente del cúbito sobre el radio, presentando todos ellos limitación de la pronosupinación de más de $25^{\circ 6}$. Sin embargo, el $80 \%$ (20 casos) retornó a sus actividades laborales previas. Strehle y Gerber realizaron otra evaluación retrospectiva de 19 lesiones de Galeazzi operadas con fijación interna del radio y reducción secundaria del cúbito con un seguimiento promedio de 83 meses (mínimo: 12 meses) ${ }^{9}$. Se incluyeron en la serie pacientes con lesiones abiertas o lesiones asociadas, algunos de los cuales requirieron además una reducción abierta RCD. Los autores no observaron diferencias significativas en la movilidad y función entre el miembro lesionado y el contralateral sano, incluso en los casos en los que no se logró una 


\section{Tabla V - EvALUACIÓN TOMOGRÁFICA DE INESTABILIDAD SEGÚN LOS CRITERIOS DE MINO ${ }^{15,} 16$ EN LAS TRES POSICIONES DE LA MUÑECA (PRONACIÓN MÁXIMA, NEUTRO, Y SUPINACIÓN MÁXIMA)}

\begin{tabular}{|c|c|c|c|c|c|c|}
\hline \multirow{3}{*}{ CASO } & \multicolumn{6}{|c|}{ SUBLUXACIÓNCUBITAL(\%)* } \\
\hline & \multicolumn{2}{|c|}{ PRONACIÓN } & \multicolumn{2}{|c|}{ NEUTRO } & \multicolumn{2}{|c|}{ SUPLNACIÓN } \\
\hline & lesionada & sama & lesionada & Sana & Lesionada & sana \\
\hline 2 & +7 & -3 & +13 & 0 & +21 & +9 \\
\hline 3 & -7 & -15 & 0 & -12 & +14 & +3 \\
\hline 4 & -5 & -5 & +9 & +9 & +14 & +11 \\
\hline 7 & -23 & -19 & -6 & -2 & 0 & +3 \\
\hline 8 & 0 & -12 & 0 & -4 & +12 & +4 \\
\hline
\end{tabular}

- Valor pesitivo = subluxación palmar, valor negative = subluxación dorsal.

reducción anatómica. Al mismo tiempo, 4 pacientes presentaron inestabilidad RCD en la evaluación clínica, asintomática sólo en uno de ellos.

En nuestro trabajo se evaluaron solamente aquellos pacientes en los cuales se logró una buena reducción del radio, con un seguimiento mínimo de 6 años. En todos los pacientes se observó clínicamente signo de la tecla, pero sólo uno presentó dolor RCD a la compresión de la articulación. Radiográficamente, en 2 casos se pudo determinar una subluxación dorsal del cúbito. Sin embargo, como se describe en la literatura, resulta muy difícil evaluar la subluxación cubital a partir de radiografías en el mismo perfil estricto de ambas muñecas ${ }^{15-19}$. En la búsqueda por lograr una determinación más objetiva y dinámica de la inestabilidad RCD, se realizó una evaluación mediante tomografías comparativas en 3 posiciones de la muñeca: neutro y prono-supinación máxima. Tomográficamente, no se observó subluxación del cúbito en ninguno de los 5 casos evaluados, incluidos los 2 pacientes que presentaron una subluxación dorsal aparente en las radiografías. Por otro lado, se observaron signos de artrosis moderada solamente en 2 casos, de los cuales, uno era una paciente de 79 años con el mismo grado de artrosis en la muñeca contralateral sin lesión previa, por lo que se la puede considerar artrosis primaria. Como describen Katolik y Trumble ${ }^{20}$, luego de la reducción secundaria de la articulación RCD, la inestabilidad crónica no se desarrolla espontáneamente, y los síntomas que los pacientes refieren inicialmente decrecen en el transcurso del tiempo, tornándose en muchos casos tolerables. En esta serie, después de más de 8 años de seguimiento promedio, un solo pa- 
Tabla VI - Evaluación RADIOLÓGICA DE LA ARTROSIS RCD

\begin{tabular}{|c|c|c|c|c|c|c|c|c|c|c|}
\hline \multirow{2}{*}{ CASO } & \multicolumn{2}{|c|}{ Osteofitos } & \multicolumn{2}{|c|}{$\begin{array}{c}\text { Pinzamiento } \\
\text { Articular }\end{array}$} & \multicolumn{2}{|c|}{$\begin{array}{l}\text { Esclerosis } \\
\text { Subcondral }\end{array}$} & \multicolumn{2}{|c|}{$\begin{array}{l}\text { Labio } \\
\text { Cubital }\end{array}$} & \multirow{2}{*}{$\begin{array}{c}\text { Estiloides } \\
\text { Cubital }\end{array}$} & \multirow{2}{*}{$\begin{array}{c}\text { Clasificación } \\
\text { Knirky Jupiter } \\
(\boldsymbol{R x} / \mathrm{TAC})\end{array}$} \\
\hline & $L$ & $s$ & $L$ & $s$ & $L$ & $s$ & $\boldsymbol{L}$ & $s$ & & \\
\hline 1 & Si & $\mathrm{Si}$ & $\mathrm{Si}$ & Si & $\mathrm{Si}$ & Si & $\mathrm{Si}$ & Si & - & II $/$ II \\
\hline 2 & No & No & No & No & No & No & No & No & Calcificación & $0 / \mathrm{I}$ \\
\hline 3 & No & No & No & No & No & No & No & No & Seudoartrosis & $0 / 0$ \\
\hline 4 & No & No & No & No & No & No & No & No & Seudoartrosis & $0 / 0$ \\
\hline 5 & Si & No & No & No & No & No & No & No & Seudoartrosis & $1 /=$ \\
\hline 6 & No & No & No & No & No & No & No & No & Calcificación & $0 /$. \\
\hline 7 & No & No & No & No & No & No & No & No & Calcificación & $0 / 1$ \\
\hline 8 & No & No & No & No & No & No & No & No & - & $0 / 1$ \\
\hline 9 & Si & No & $\mathrm{Si}$ & No & Si & No & $\mathrm{Si}$ & No & - & II $/$ - \\
\hline
\end{tabular}

ciente presentó síntomas leves, y todos los pacientes evaluados presentaron un rango de movilidad funcional que les permitió retornar a sus actividades diarias y laborales previas. Además, no se observó una correlación entre inestabilidad y artrosis RCD.

En un estudio cadavérico, Moore et al..$^{21}$ concluyen que si el acortamiento inicial del radio es mayor a $5 \mathrm{~mm}$ debe existir lesión del complejo fibrocartílago triangular o de la membrana interósea, y que si el acortamiento radial supera los $10 \mathrm{~mm}$, debe existir una disrupción de ambas estructuras. En nuestra serie el acortamiento inicial mínimo del radio fue de $14 \mathrm{~mm}$, por lo cual asumimos que en todos los casos el complejo fibrocartílago triangular se encontraba lesionado por el trauma. En todos ellos se logró una adecuada restauración de la longitud radial en el postoperatorio y en el transcurso de su evolución (diferencias no significativas), posibilitando la cicatrización ligametaria.
Las fracturas-luxaciones de Galeazzi ocurren con mayor frecuencia en hiperpronación del antebrazo acompañado de una subluxación dorsal del cúbito $^{9}$, resultando más probablemente en rupturas periféricas del complejo fribrocartílago triangular (lesiones tipo 1B, 1C, y $1 \mathrm{D}$ de Palmer $)^{22}$. La buena vascularización periférica del fibrocatílago triangular podría explicar la posibilidad de cicatrización de estas lesiones luego de una adecuada reducción de la articulación radiocubital distal ${ }^{23,24}$. No se ha demostrado una correlación entre lesiones del fibrocartílago triangular y la presencia de dolor en la muñeca. De todas maneras, resulta interesante que los 6 casos de nuestra serie que presentaron seudoartrosis o calcificaciones en la estiloides cubital se encontraron asintomáticos en la evaluación final.

Una vez reducido y fijado el radio resulta fundamental examinar cuidadosamente la reducción y la estabilidad RCD, comparándolas siem- 
pre con la muñeca contralateral ${ }^{25}$. De acuerdo con Rettig et a., para mantener una precisa reducción del cúbito sobre la fosa sigmoidea es necesaria la fijación de la RCD con una aguja, evitando así la subluxación persistente del cúbito $^{8}$. Mestdagh et al. concluyen que la inmovilización en supinación del antebrazo por 4-6 semanas sin la fijación adicional con una aguja sería suficiente ${ }^{6}$. En un estudio reciente, Rothe et al. observaron que 8 de 13 pacientes en los cuales se realizó una fijación transitoria con agujas RCD presentaron limitación de la prono-supinación ${ }^{26}$. En la serie actual se bloqueó en todos los casos la prono-supinación del antebrazo con un yeso braquiopalmar en posición neutra o supinación moderada por 3 semanas, con buenos resultados alejados. Sin embargo, debido a la naturaleza retrospectiva de todos estos estudios, resulta difícil concluir si la fijación adicional con agujas resulta superior a la inmovilización con yeso.

En conclusión, los resultados obtenidos en este trabajo sugieren que el pronóstico clínico y radiológico a largo plazo de la articulación RCD en lesiones de Galeazzi es favorable cuando se logra una adecuada reducción secundaria luego de la reducción y fijación interna del radio. En estos casos no sería necesaria la reparación quirúrgica del complejo fibrocartílago triangular. La reducción abierta de la articulación RCD estaría indicada exclusivamente cuando no es posible lograr una buena reducción indirecta por interposición de partes blandas.

\section{BIBLIOGRAFÍA}

1. Cooper Sir AP. Treatise of Dislocations and Fractures of the Joints. London: Longman, Hurst, Rees, Orme, Brown \& Cox, 1822.

2. Reckling FW, Peltier LF. Riccardo Galeazzi and Galeazzi's fracture. Surgery, 1965; 58: 453-60.

3. Mikic ZD. Galeazzi fracture-dislocations. J Bone Joint Surg Am, 1975 ; 57: 1071-80.

4. Moore TM, Klein JP, Patzakis MJ, et al. Results of compression-plating of closed Galeazzi fractures. J Bone Joint Surg AM, 1985; 67: 1015-21.

5. Graham TJ. Surgical Correction of malunited fractures of the distal end of the radius with plates. J Am Acad Orthop Surg, 1997; 5: 270-281.

6. Mestdagh $\mathrm{H}$, Duquennoy $\mathrm{A}$, Letendart $\mathrm{J}$, et al. Long-term results in the treatment of fracture-dislocation of Galeazzi in adults. Report of twenty-nine cases. Ann Chir Main, 1983; 2: 125-33.

7. Mohan K, Gupta AK, Sharma J, et al. Internal fixation in 50 cases of Galeazzi fracture. Acta Orthop Scand, 1988; 59: 314-8.
8. Rettig ME, Raskin KB; Galeazz fracture-dislocation: A new treatment oriented classification. $\mathrm{J}$ Hand Surg Am, 2001; 26: 228-35.

9. Strehle J, Gerber C. Distal radioulnar joint function after $\mathrm{Ga}$ leazzi fracture dislocations treated by open reduction and internal fixation. Clin Orthop, 1993; 293: 240-5.

10. Crosby CA, Whebe MA, Mawr B. Hand strength: normative values. J Hand Surg Am, 1994; 19: 665-70.

11. Rosales RS, Benseny Delgado E, Díez de la Lastra-Bosch I. Evaluation of the Spanish version of the DASH and carpal tunnel syndrome health-related quality of life Instruments: Cross-cultural adaptation process and reliability. J Hand Surg Am, 2002; 27: 334-43.

12. Adams BD. Inestabilidad de la articulación radio cubital inferior. Green Cirugía de la Mano. DP. Green (Ed). Vol. 1. Edición en español. Madrid: Marbán SL, 2007: 609-10.

13. Steyers CM, Blair WF. Measuring ulnar variance: a comparison of techniques. J Hand Surg Am, 1989; 14: 607-12.
14. Knirk JL, Jupiter J. Intra-articular fractures of the distal end of the radius in young adults. $\mathrm{J}$ Bone Joint Surg Am, 1986; 68: 647-59.

15. Mino DE, Palmer AK, Levinsohn EM. The role of radiography and computarized tomography in the diagnosis of subluxation and discolation of the distal radioulnar joint. $J$ Hand Surg Am, 1983; 8: 2331.

16. Mino DE, Palmer AK, Levinsohn EM. Radiography and computarized tomography in the diagnosis of incongruity of distal radio-ulnar joint. $\mathrm{J}$ Bone Joint Surg Am, 67: 247-52.

17. Lo KY, MacDermid JC, Bennett JD, et al.: The radioulnar ratio: a new method of quantifying distal radioulnar joint subluxation. J Hand Surg Am, 2001; 26: $236-43$.

18. Rozental TD, Beredjiklian PK, Bozentka DJ. Instability of the distal radioulnar joint: current diagnostic and treatment methods. Curr Orthop, 2003; 14: 245-51.

19. Wechsler RJ, Wehbe MA, Rifkin MD, et al.. Computed tomography diagnosis of distal 
radioulnar subluxation. Skeletal Radiol 1987; 16: 1-5.

20. Katolik L, Trumble T. Distal radioulnar joint dysfunction. J Am Soc Surg Hand, 2005; 5: 8-29.

21. Moore TM, Lester DK, Sarmiento A. The stabilizing effect of soft-tissue constraints in artificial Galeazzi fractures. Clin Orthop, 1985; 194: 189 -94.

22. Palmer AK. Triangular fibrocar- tilage complex lesions: A classification. J Hand Surg Am, 1989; 14: 594-601.

23. Dell PC. Distal radioulnar joint dysfunction. Hand Clin, 1987; 3: 563-7.

24. Thiru-Pathi RG, Ferlic DC, Clayton ML, et al. Arterial anatomy of the triangular fibrocartilage of the wrist and its surgical significance. J Hand Surg Am, 1986; 11: 258-63.
25. Reckling FW. Unstable Fracture Dislocations of the forearm (Monteggia and Galeazzi Lesions). J Bone Joint Surg Am, 1982; 64: 857-63.

26. Rothe M, Rudy T, Stankovic P, et al. Treatment of Galeazzi's fracture - is the surgical revision of the radioulnar joint necessary? Handchir Mikrochir Plast Chir, 2001; 33: 252-7. 